\title{
Частота гипопитуитаризма
}

\section{у пациентов с макроаденомами гипофиза с различной гормональной активностью}

\author{
Кривошеева Ю.Г. • Иловайская И.А.
}

Актуальность. Частота распространения опухолей гипофиза, согласно данным последних эпидемиологических исследований, составляет 1 случай на 865-2688 человек взрослого населения. Сведения о частоте развития гипопитуитаризма при макроаденомах гипофиза существенно варьируют - в диапазоне от 37 до 85\% среди впервые выявленных гормонально-неактивных макроаденом гипофиза (ГНАГ). Информация о частоте гипопитуитаризма в когорте впервые выявленных макроаденом с пролактинсекретирующей и соматотропинсекретирующей активностью практически отсутствует.

Цель - оценить частоту гипопитуитаризма у пациентов с макроаденомами гипофиза с различной гормональной активностью и выявить возможные прогностические факторы его развития.

Материал и методы. Проанализированы данные 293 пациентов с макроаденомами гипофиза с различной гормональной активностью: ГНАГ $(n=121)$, пролактиномами $(n=59)$ и соматотропиномами $(n=113)$. Пациенты были обследованы до проведения какого-либо лечения.

Результаты. Частота выявления гипопитуитаризма составила 59/121 (39,9\%) среди пациентов с гормонально-неактивными опухолями гипофиза, 18/59 (27,3\%) среди пролактином и 19/113 (14\%) среди соматотропином $(p<0,001)$. Признаки компрессии хиазмы, а также наличие хиазмального синдрома статистически значимо чаще встречались у пациентов с гипопитуитаризмом по сравнению с пациентами без этого состояния во всех обследованных подгруппах. Относительный риск развития гипопитуитаризма при наличии компрессии хиазмы составил для ГНАГ 2,10 (95\% доверительный интервал (ДИ) 1,50-2,95, $p=0,003)$, для пролактином 1,667 (95\% ДИ 1,29-2,18, $\mathrm{p}=0,005$ ), для соматотропином 1,45 (95\% дИ 1,56-2,48, $\mathrm{p}=0,001)$. Относительный риск развития гипопитуитаризма при хиазмальном синдроме составил для ГНАГ 1,66 (95\% ДИ 1,26-2,18, p=0,009), для пролактином 2,08 (95\% ДИ 1,60-2,69, $\mathrm{p}=0,001)$, для соматотропином 1,97 (95\% ДИ $1,56-2,48, p=0,005)$. В подгруппе ГНАГ наиболее часто встречался гипотиреоз - 36/59 (61,0\%), в подгруппе пролактином - вторичный гипогонадизм, 34/59 (57,6\%), однако формирование гипогонадизма служит одним из проявлений стойкой патологической гиперпролактинемии, которая характерна для пролактином; в подгруппе соматотропином вторичный гипотиреоз выявлен у 11/19 (57,9\%) пациентов.
Заключение. Выявление гипопитуитаризма до какого-либо лечения зависело от типа гормональной активности опухоли гипофиза и с наибольшей частотой встречалось при ГНАГ. Факторами развития гипопитуитаризма были наличие компрессии хиазмы, наличие хиазмального синдрома, вертикальный размер опухоли гипофиза, объем опухоли гипофиза. Вторичная надпочечниковая недостаточность как наиболее опасная составляющая гипопитуитаризма встречалась наиболее редко.

Ключевые слова: макроаденомы гипофиза, гормонально-неактивные опухоли гипофиза, пролактиномы, соматотропиномы, гипопитуитаризм

Для цитирования: Кривошеева ЮГ, ИловайСКая ИА. Частота гипопитуитаризма у пациентов с макроаденомами гипофиза с различной гормональной активностью. Альманах клинической медицины. 2021;49(4):261-267. doi: 10.18786/20720505-2021-49-047.

Поступила 20.10.2021; доработана 29.10.2021; принята к публикации 03.11.2021; опубликована онлайн 10.11.2021
บ астота распространения опухолей гипофиза, согласно данным последних эпидемиологических исследований, составляет 1 случай на 865-2688 человек взрослого населения [1-3]. Наиболее часто встречающиеся опухоли - пролактиномы (32-66\% от всех опухолей гипофиза), гормонально-неактивные (15-54\%) и соматотропиномы (8-16\%) [1]. Доли микро- и макроаденом гипофиза в целом схожи и составляют примерно по $50 \%$ [1], хотя могут варьировать в зависимости от типа гормональной активности опухоли гипофиза: доля макроаденом среди соматотропином достигает 75-80\%, тогда как среди пролактином - $40-45 \%[1,4,5]$.
Макроаденомы гипофиза представляют собой более грозное состояние, чем микроаденомы, за счет сдавления окружающих структур головного мозга, включая ножку гипофиза и перекрест зрительных нервов, что может стать причиной головных болей, нарушения зрения и развития гипопитуитаризма $[6,7]$.

Сведения о частоте гипопитуитаризма при макроаденомах гипофиза часто ограничиваются гормонально-неактивными макроаденомами, которые составляют от 37 до 85\% [7-12]. Данные о частоте гипопитуитаризма среди впервые выявленных макроаденом с пролактинсекретирующей и СТГ-секретирующей активностью еще более малочисленны [4]. Гипопитуитаризм 
(уменьшение продукции тропных гормонов гипофиза с последующим снижением функции соответствующих периферических эндокринных желез) часто не имеет ярких клинических проявлений; жалобы могут варьировать в зависимости от вида тропной недостаточности, но на начальных этапах заболевания часто неспецифичны и сводятся к общей утомляемости, слабости, снижению толерантности к физическим нагрузкам [13]. Однако без лечения состояние будет прогрессивно ухудшаться, что может осложнить наблюдение больного и/или исход лечения. Кроме того, гипопитуитаризм признан фактором повышенной смертности пациентов $[8,14]$. Именно поэтому очень важно понимать частоту встречаемости и факторы развития гипопитуитаризма среди макроаденом гипофиза для своевременной диагностики и назначения заместительной терапии.

Цель - оценить частоту гипопитуитаризма у пациентов с макроаденомами гипофиза с различной гормональной активностью и выявить его возможные прогностические факторы.

\section{Материал и методы}

В одномоментное исследование вошли пациенты, обследованные в ГБУЗ МО МОНИКИ им. М.Ф. Владимирского с 2004 по 2018 г. Были проанализированы данные историй болезни и амбулаторных карт 293 пациентов с макроаденомами гипофиза с различной гормональной активностью, среди которых были гормонально-неактивные макроаденомы гипофиза (ГНАГ, $\mathrm{n}=121)$, пролактиномы $(\mathrm{n}=59)$ и соматотропиномы $(\mathrm{n}=113)$. Пациенты были обследованы до проведения какого-либо лечения. Характеристика пациентов, включенных в исследование, дана в табл. 1.

Магнитно-резонансную томографию (МРТ) головного мозга с прицельным исследованием гипофиза выполняли на высокопольном аппарате Intera Achieva (Philips) со сверхсильной напряженностью магнитного поля 3,0 Тл, внутривенным введением внеклеточных гадолинийсодержащих контрастных препаратов. Оценивали максимальный линейный размер (в миллиметрах) в вертикальной, сагиттальной (передне-задней) и фронтальной (поперечной) плоскостях. Для вычисления объема опухоли гипофиза использовали следующую формулу: АВС $\times \pi \times \frac{4}{3}$, где АВС размеры полуосей (сагиттальной, вертикальной и фронтальной) опухоли. Характеризовали частоту и характер распространения опухоли за пределы турецкого седла.
Кривошеева Юлия Григорьевна - науч. сотр. отделения терапевтической эндокринологии'; ORCID: https://orcid.org/00000002-2061-5235 $\triangle 129110$, г. Москва, ул. Щепкина, 61/2, Российская Федерация. Тел.: +7 (916) 4706041. E-mail: yulya.k.89@inbox.ru Иловайская Ирэна Адольфовна - д-р мед. наук, доцент, вед. науч. сотр. отделения терапевтической эндокринологии, профессор курса частной эндокринологии кафедры эндокринологии факультета усовершенствования врачей'; ORCID: https:// orcid.org/0000-00033261-7366.

Тел.: +7 (916) 6772645 . E-mail: irena.ilov@yandex.ru

' ГБУЗ МО «Московский областной научно-исследовательский клинический институт им. М.Ф. Владимирского»; 129110, г. Москва, ул. Щепкина, 61/2, Российская Федерация
Пациентам выполняли гормональное обследование: определение уровней мономерного пролактина, инсулиноподобного фактора роста-1 (ИФР-1), соматотропного гормона (СТГ), тиреотропного гормона, свободного тироксина, лютеинизирующего гормона, фолликулостимулирующего гормона, эстрадиола у женщин и общего тестостерона у мужчин, адренокортикотропного гормона и кортизола утром, по показаниям - кортизола в ходе малой дексаметазоновой пробы. Гормональные обследования проводили в сыворотке венозной крови натощак иммуноферментными методами на анализаторе Immulite 2000 (Diagnostic Products Corporation, Лос-Анджелес, США).

Помимо характерных клинических симптомов диагностическими критериями соматотропиномы были значимое повышение уровня ИФР-1 и отсутствие подавления концентрации СТГ менее 1 нг/мл в ходе теста с нагрузкой глюкозой; диагностическим критерием пролактиномы было значимое (более чем в 4 раза от верхней границы референсных значений) повышение уровня пролактина; гормонально-неактивными считались аденомы гипофиза у пациентов с нормальным уровнем ИФР-1 и повышением уровня пролактина не более чем в 2 раза от верхней границы референсных значений.

Диагностическими критериями гипопитуитаризма служили: вторичный гипотиреоз - уровень тиреотропного гормона не выше верхней границы референсных значений в сочетании со сниженным уровнем свободного тироксина; вторичный гипогонадизм - сниженные или низконормальные концентрации гонадотропинов в сочетании со снижением концентрации периферических половых стероидов, вторичный гипокортицизм - концентрация кортизола в 8 утра менее 250 нмоль/л в сочетании с уровнем адренокортикотропного гормона менее 5 пг/мл (референсные значения до 46 пг/мл).

Пациенты были осмотрены офтальмологом, проводились осмотр глазного дна (офтальмоскоп Heine Beta 200), периметрия (периметр Oculus Centerfield plus \# SN 0801 8960). Критериями хиазмального синдрома считали наличие 3 клинических признаков: атрофии зрительного нерва, сужения полей зрения, снижения остроты зрения.

Исследование проведено в рамках диссертационной работы «Гормональные нарушения у пациентов с макроаденомами гипофиза», одобрено независимым комитетом по этике № 1 от 22.01.2015. Все пациенты подписали информированное согласие. 
Таблица 1. Характеристика пациентов с макроаденомами гипофиза различной гормональной активности

\begin{tabular}{|c|c|c|c|}
\hline \multirow[t]{2}{*}{ Показатель } & \multicolumn{3}{|l|}{ Группа } \\
\hline & $\begin{array}{l}\text { гормонально- } \\
\text { неактивные } \\
\text { аденомы } \\
\text { гипофиза } \\
(\mathrm{n}=121)\end{array}$ & $\begin{array}{l}\text { пролактиномы } \\
(n=59)\end{array}$ & $\begin{array}{l}\text { соматотропиномы } \\
(\mathrm{n}=113)\end{array}$ \\
\hline $\begin{array}{c}\text { Возраст пациентов (Mе } \\
\text { [Q25; Q75]), годы }\end{array}$ & $59[50 ; 64,5]$ & $32[27 ; 48,5]$ & $53[41 ; 60]$ \\
\hline $\begin{array}{l}\text { Соотношение } \\
\text { женщин/мужчин, \% }\end{array}$ & $63,5 / 36,5$ & $65 / 35$ & $80 / 20$ \\
\hline
\end{tabular}

Критериями включения в исследование были наличие опухоли гипофиза с хотя бы одним размером более 10 мм и диагноз соматотропиномы, пролактиномы или гормонально-неактивной опухоли гипофиза после проведенного гормонального обследования.

Критериями исключения были отсутствие подавления уровня кортизола в ходе малой дексаметазоновой пробы (уровень кортизола более 50 нмоль/л) и невозможность дифференциальной диагностики гормонально-неактивной опухоли гипофиза с гиперпролактинемией и пролактиномой.

Статистический анализ проводили с использованием программы Statistica 13.2 (Dell inc., США). Гипотезу о нормальности распределения количественных переменных проверяли с помощью критерия Шапиро - Уилка. Условия нормальности распределения и равенства дисперсий не выполнялись для исследуемых переменных, поэтому использовали критерии для непараметрических данных. Для количественных переменных рассчитывали медианы и квартили. Сравнение трех групп проводилось с помощью критерия Краскела - Уоллиса с дальнейшим апостериорным анализом с поправкой на множественные сравнения. Анализ качественных данных проводили с помощью точного критерия Фишера с поправкой Бонферрони при множественных попарных сравнениях. Для определения чувствительности и специфичности отрезных точек использовали ROC-анализ. Для определения статистической значимости различий категориальных переменных в независимых группах применяли тест хи-квадрат $\left(\chi^{2}\right)$. Критический уровень значимости при статистической проверке гипотез в данном исследовании был принят равным 0,05. Относительный риск рассчитывали при помощи онлайн-калькулятора (https://medstatistic.ru/ calculators/calcrisk.html).

\section{Результаты}

Сравнительный анализ половозрастных характеристик пациентов и особенности опухолей гипофиза в зависимости от наличия/отсутствия гипопитуитаризма представлены в табл. 2-4.

Распределение пациентов по полу было сравнимо в подгруппах с пролактиномами и соматотропиномами, но не с ГНАГ, - доля мужчин с гипопитуитаризмом была выше (см. табл. 2). Относительный риск развития гипопитуитаризма у больных с ГНАГ мужского пола составил 1,575 (95\% доверительный интервал (ДИ) 1,2122,047). Статистически значимых различий возраста пациентов с гипопитуитаризмом и без такового выявлено не было (см. табл. 2-4).

Частота компрессии хиазмы по данным МРТ зависела от типа гормональной активности опухоли гипофиза, различия были статистически значимыми $(\mathrm{p}<0,001)$. Признаки компрессии хиазмы по данным МРТ, а также хиазмального синдрома статистически значимо чаще встречались у пациентов с гипопитуитаризмом по сравнению с пациентами без этого состояния во всех обследованных подгруппах (см. табл. 2-4). Относительный риск развития гипопитуитаризма при наличии компрессии хиазмы составил для ГНАГ 2,10 (95\% ДИ 1,50-2,95, p=0,003), для

Таблица 2. Характеристики гормонально-неактивных опухолей гипофиза в зависимости от наличия/отсутствия гипопитуитаризма

\begin{tabular}{|c|c|c|c|}
\hline Показатель & Гипопитуитаризм есть $(n=59)$ & Гипопитуитаризма нет (n=62) & Значение $p$ \\
\hline Соотношение женщин/мужчин, \% & $57,6 / 42,4$ & $79 / 21$ & 0,012 \\
\hline Возраст пациентов (Ме [Q25; Q75]), годы & $59[50 ; 66,5]$ & $57[50 ; 64]$ & 0,666 \\
\hline Признаки компрессии хиазмы по данным МРТ, \% & 72,4 & 38,3 & $<0,001$ \\
\hline Наличие хиазмального синдрома, \% & 52,5 & 27,4 & 0,011 \\
\hline Вертикальный размер (Me [Q25; Q75]), мм & $25[19 ; 33,3]$ & $17,9[12,8 ; 24,3]$ & $<0,001$ \\
\hline Объем опухоли (Ме [Q25; Q75]), мм³ & $6119,5[3118,5 ; 12375]$ & $2345[1155 ; 5945,3]$ & $<0,001$ \\
\hline
\end{tabular}

МРТ - магнитно-резонансная томография 
Таблица 3. Характеристики пролактином в зависимости от наличия/отсутствия гипопитуитаризма

\begin{tabular}{llll}
\hline Показатель & Гипопитуитаризм есть (n=18) & Гипопитуитаризма нет $(\mathrm{n}=41)$ & 3начение $p$ \\
\hline Соотношение женщин/мужчин, \% & $67 / 33$ & $70,7 / 29,3$ & 0,991 \\
Возраст пациентов (Ме [Q25; Q75]), годы & $43[31,5 ; 54]$ & $30[24 ; 42,3]$ & 0,086 \\
Признаки компрессии хиазмы по данным МРТ, \% & 50 & 25 & $<0,001$ \\
Наличие хиазмального синдрома, \% & 50 & 14,6 & $<0,001$ \\
Вертикальный размер (Ме [Q25; Q75]), мм & $22,7[18,5 ; 30]$ & $17[12 ; 24]$ & 0,029 \\
Объем опухоли (Ме [Q25; Q75]), мм³ & $7296[3093,75 ; 11272,5]$ & $2490[1010,6 ; 7784]$ & 0,022 \\
\hline
\end{tabular}

МРТ - магнитно-резонансная томография

Таблица 4. Характеристики соматотропином в зависимости от наличия/отсутствия гипопитуитаризма

\begin{tabular}{llll}
\hline Показатель & Гипопитуитаризм есть (n=19) & Гипопитуитаризма нет (n=94) & 3начение $p$ \\
\hline Соотношение женщин/мужчин, \% & $84,2 / 15,8$ & $75,5 / 24,5$ & 0,414 \\
Возраст пациентов (Ме [Q25; Q75]), годы & $42[29 ; 54]$ & $54[43,5 ; 62]$ & 0,119 \\
Признаки компрессии хиазмы по данным МРТ, \% & 26,3 & 12,6 & 0,021 \\
Наличие хиазмального синдрома, \% & 31,6 & 6,4 & $<0,001$ \\
Вертикальный размер (Ме [Q25; Q75]), мм & $19,5[15,75 ; 28,18]$ & $15[12 ; 22]$ & 0,028 \\
Объем опухоли (Ме [Q25; Q75]), мм³ & $4127,17[1851,75 ; 16291,8]$ & $2394[1102,9 ; 4324]$ & 0,043 \\
\hline
\end{tabular}

МРТ - магнитно-резонансная томография

пролактином 1,667 (95\% ДИ 1,29-2,18, p=0,005), для соматотропином 1,45 (95\% ДИ 1,56-2,48, $\mathrm{p}=0,001)$. Наличие хиазмального синдрома также было важным предиктором гипопитуитаризма, относительный риск развития последнего при данном синдроме составил для ГНАГ 1,66 (95\% ДИ 1,26-2,18, p=0,009), для пролактином 2,08 (95\% ДИ 1,60-2,69, p=0,001), для соматотропином 1,97 (95\% ДИ 1,56-2,48, p=0,005).

Вертикальный размер опухоли и ее объем также были статистически значимо больше у пациентов с гипопитуитаризмом (см. табл. 2-4). Вертикальный размер опухоли более 22,5 мм (площадь под ROC-кривой (англ. area under curve, AUC) $0,7209, \mathrm{p}<0,0001)$ и объем опухоли более 4472 мм $^{3}$ (AUC ROC-кривой 0,7066, p<0,0001) были статистически значимыми отрезными точками для наличия гипопитуитаризма, хотя и с невысокими чувствительностью и специфичностью (чувствительность 55,91 и 62,77\%, специфичность 73,44 и 70,16\% соответственно).

Доля различных видов гипофизарных недостаточностей в обследованных подгруппах пациентов варьировала в зависимости от вида опухоли гипофиза. В подгруппе ГНАГ наиболее часто встречался гипотиреоз. Его частота составила 36/59 (61,0\%); частота вторичного гипогонадизма и вторичного гипокортицизма была 31/59 (52,5\%) и 26/59 (44,1\%) соответственно (рис. 1 А).

В подгруппе пролактином с наибольшей частотой встречался вторичный гипогонадизм 34/59 (57,6\%), однако формирование гипогонадизма считается одним из проявлений стойкой патологической гиперпролактинемии, которая характерна для пролактином. В рамках настоящего исследования было невозможно оценить, был ли гипогонадизм следствием гиперпролактинемии или «масс-эффекта» опухоли, поэтому мы рассматривали пациентов с изолированным гипогонадизмом на фоне значительной гиперпролактинемии как неимеющих гипопитуитаризм. Таким образом, в подгруппу больных с пролактиномами и гипопитуитаризмом вошли 18 больных, у которых кроме гипогонадизма отмечался вторичный гипотиреоз (18/18, 100\%), из них у 2 был дополнительно диагностирован вторичный гипокортицизм (2/18, 11,1\%); случаев изолированного вторичного гипокортицизма в этой подгруппе не регистрировали (рис. 1Б).

В подгруппе соматотропином, как и среди пролактином, наиболее часто встречался вторичный гипогонадизм - в 14/19 (73,7\%) случаев, однако у 4 пациенток был изолированный центральный гипогонадизм на фоне гиперпролактинемии. Вторичный гипогонадизм на фоне нормального 
А Гормонально-неактивные опухоли гипофиза

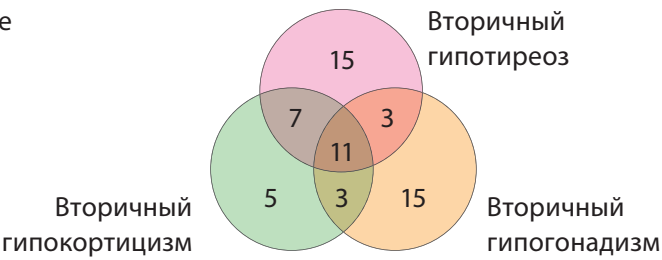

[ Пролактиномы

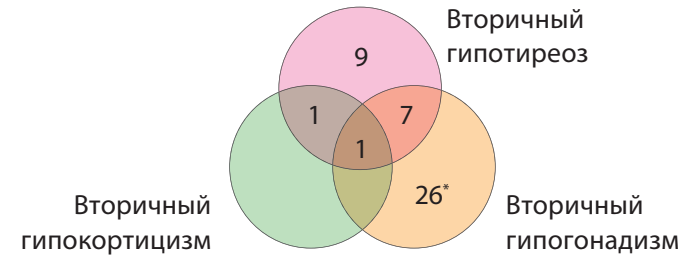

В Соматотропиномы

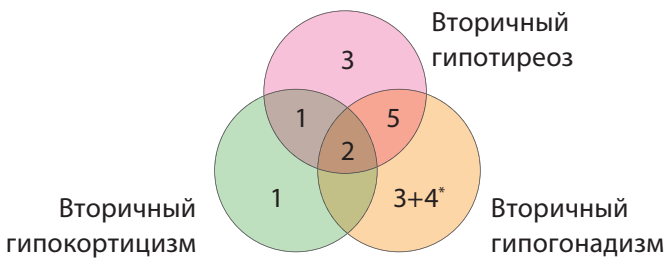

Распределение видов тропных недостаточностей у пациентов с макроаденомами гипофиза с различной гормональной активностью; "пациенты с гиперпролактинемией, не учитывались при подсчете частоты гипопитуитаризма

уровня пролактина выявлен у $10 / 19(52,6 \%)$, вторичный гипотиреоз - у 11/19 (57,9\%), вторичная надпочечниковая недостаточность - у 4/19 (21,0\%) больных (рис. 1B).

\section{Обсуждение}

В целом гипопитуитаризм выявлен у 96 из 293 (32,8\%) обследованных пациентов с макроаденомами гипофиза. Частота выявления гипопитуитаризма различалась в подгруппах пациентов с различной гормональной активностью и составила 59/121 (48,8\%) в подгруппе ГНАГ, 18/59 (30,5\%) в подгруппе пролактином и 19/113 (16,8\%) в подгруппе соматотропином $(\mathrm{p}<0,001)$. Относительный риск развития гипопитуитаризма был в 1,452,1 раза выше при признаках компрессии хиазмы по данным МРТ и в 1,66-2,08 раза выше при наличии хиазмального синдрома. При ГНАГ риск развития гипопитуитаризма был в 1,58 раза выше у мужчин. Вертикальный размер опухоли гипофиза более 22,5 мм и объем опухоли гипофиза более 4472 мм $^{3}$ также указывали на вероятное наличие гипопитуитаризма.

Частота гипопитуитаризма при ГНАГ в нашей когорте пациентов соответствует данным, полученным в аналогичных исследованиях $[9,11,12]$.
Статистически значимые различия по возрасту пациентов в нашем исследовании получены не были.

Частота компрессии хиазмы по данным МРТ зависела от типа гормональной активности опухоли гипофиза и варьировала от 26,3 до $72,4 \%$ ( $\mathrm{p}<0,001)$, частота хиазмального синдрома была несколько ниже $(31,6-52,5 \%)$ и также зависела от типа гормональной активности $(\mathrm{p}=0,005)$. В нашем исследовании частота хиазмального синдрома не коррелировала с частотой признаков компрессии хиазмы, что совпадает с данными работы I.H. Lee и соавт., где частота компрессии хиазмы по результатам МРТ также превалировала над зрительными нарушениями - 84,8 против 42,6\% [15]. Тем не менее и наличие компрессии хиазмы, и наличие хиазмального синдрома выступали независимыми прогностическими факторами развития гипопитуитаризма.

В работе D. Margaritopoulos и соавт. была схожая когорта пациентов с ГНАГ $(\mathrm{n}=148)$, при этом гипопитуитаризм встречался у 66,2\% пациентов, что несколько чаще, чем в настоящем исследовании $(48,8 \%)$. Факторами развития гипопитуитаризма были более старший возраст пациентов, максимальный размер опухоли гипофиза, компрессия хиазмы [9]. В нашей работе пациенты с гипопитуитаризмом и без такового не различались по возрасту, однако другие факторы были аналогичными.

Ограничения исследования. В нашей работе невозможно было оценить частоту СТГнедостаточности в связи с отсутствием достаточного количества данных в анализируемой медицинской документации в группах с ГНАГ и пролактиномами. В подгруппах пролактином и соматотропином были пациенты с изолированным гипогонадизмом на фоне гиперпролактинемии (в подгруппе ГНАГ таких пациентов не было). Отличить гиперпролактинемический гипогонадизм от вторичного гипогонадизма вследствие «масс-эффекта» опухоли гипофиза возможно после нормализации уровня пролактина, однако это не входило в задачи данного исследования. Именно поэтому эти больные не были учтены как пациенты с гипопитуитаризмом. Возможно, данные ограничения несколько снизили частоту гипопитуитаризма в нашей группе.

\section{Заключение}

Гипопитуитаризм не имеет четких клинических проявлений, и для его диагностики следует проводить гормональное обследование, которое должно быть обосновано. В ходе нашей работы было показано, что обследование для исключения 
гипопитуитаризма необходимо проводить больным с макроаденомами гипофиза при наличии следующих факторов: при гормонально-неактивных аденомах гипофиза - мужской пол; вне зависимости от гормональной активности - признаки компрессии хиазмы, хиазмальный синдром, вертикальный размер опухоли более 22,5 мм, объем опухоли более $4472 \mathrm{~mm}^{3}$.

Гипопитуитаризм представляет собой жизнеугрожающее осложнение макроаденом гипофиза. При необходимости нейрохирургического лечения макроаденомы некомпенсированный гипопитуитаризм повышает анестезиологический риск и осложняет течение послеоперационного периода. Вне зависимости от выбранной тактики дальнейшего лечения макроаденомы гипофиза гипопитуитаризм без лечения значимо снижает качество жизни пациентов и сокращает ее продолжительность. Своевременная диагностика гипопитуитаризма и назначение заместительной терапии позволят избежать этих проблем при курации пациентов с опухолями гипофиза. (9)

\section{Дополнительная информация}

\section{Финансирование}

Работа проведена без привлечения дополнительного финансирования со стороны третьих лиц.

\section{Конфликт интересов}

Авторы не сообщают о конфликте интересов.

Участие авторов

Оба автора внесли равный вклад в написание статьи. Оба автора прочли и одобрили финальную версию статьи перед публикацией, согласны нести ответственность за все аспекты работы и гарантируют, что ими надлежащим образом были рассмотрены и решены вопросы, связанные с точностью и добросовестностью всех частей работы.

\section{Благодарности}

Авторы выражают благодарность научному сотруднику лаборатории медико-физических исследований ГБУз МО МОНИКИ им. М.Ф. Владимирского к.м.н. А.А. Глазкову за помощь в статистической обработке результатов, сотруднику отделения рентгеновской компьютерной и магнитно-резонансной томографии д.м.н. Г.А. Сташук и врачу-офтальмологу Ю.Н. Бардеевой за помощь в сборе материала для данной публикации.

\section{Литература/References}

1. Molitch ME. Diagnosis and Treatment of Pituitary Adenomas: A Review. JAMA. 2017;317(5): 516-524. doi: 10.1001/jama.2016.19699.

2. Chen C, Hu Y, Lyu L, Yin S, Yu Y, Jiang S, Zhou P. Incidence, demographics, and survival of patients with primary pituitary tumors: a SEER database study in 2004-2016. Sci Rep. 2021;11:15155. doi: 10.1038/s41598-021-94658-8.

3. Ntali G, Wass JA. Epidemiology, clinical presentation and diagnosis of non-functioning pituitary adenomas. Pituitary. 2018;21(2):111-118. doi: 10.1007/s11102-018-0869-3.

4. БелаяЖЕ, Голоунина ОО, РожинскаяЛЯ, Мельниченко ГА, Исаков МА, Луценко АС, Алексеева ТМ, Зенкова ТС, Пржиялковская ЕГ, Панюшкина ГМ, Илюхина ОБ, Иванова ЕИ, Кришталь ЕА, Вачугова АА, Пигарова ЕА, Дзеранова ЛК, Марова ЕИ, Арапова СД, Мамедова ЕО, Гребенникова ТА, Анциферов МБ, Древаль АВ, Дедов ИИ. Эпидемиология, клинические проявления и эффективность различных методов лечения акромегалии по данным единого российского регистра опухолей гипоталамо-гипофизарной системы. Проблемы Эндокринологии. 2020;66(1):93103. doi: 10.14341/probl10333. [Belaya ZhE, Golounina OO, Rozhinskaya LYa, Melnichenko GA, Isakov MA, Lutsenko AS, Alekseeva T, Zenkova TS, Przhiyalkovskaya EG, Panyushkina GM, Ilukhina OB, Ivanova El, Krishtal EA, Vachygova AA, Pigarova EA, Dzeranova LK, Marova El, Arapova SD, Mamedova EO, Grebennikova TA,
Antsiferov MB, Dreval AV, Dedov II. [Epidemiology, clinical manifestations and efficiency of different methods of treatment of acromegaly according to the United Russian Registry of Patients with Pituitary Tumors]. Problems of Endocrinology. 2020;66(1):93-103. Russian. doi: 10.14341/probl10333.]

5. Maione L, Chanson P. National acromegaly registries. Best Pract Res Clin Endocrinol Metab. 2019;33(2):101264. doi: 10.1016/j. beem.2019.02.001.

6. Arafah BM, Prunty D, Ybarra J, Hlavin ML, Selman WR. The dominant role of increased intrasellar pressure in the pathogenesis of hypopituitarism, hyperprolactinemia, and headaches in patients with pituitary adenomas. J Clin Endocrinol Metab. 2000;85(5):1789-1793. doi: 10.1210/jcem.85.5.6611.

7. Mercado M, Melgar V, Salame L, Cuenca D. [Clinically non-functioning pituitary adenomas: Pathogenic, diagnostic and therapeutic aspects]. Endocrinol Diabetes Nutr. 2017;64(7): 384-395. English, Spanish. doi: 10.1016/j.endinu.2017.05.009.

8. Pappachan JM, Raskauskiene D, Raman Kutty V, Clayton RN. Excess mortality associated with hypopituitarism in adults: a meta-analysis of observational studies. J Clin Endocrinol Metab. 2015;100(4):1405-1411. doi: 10.1210/jc.20143787.

9. Margaritopoulos D, Vassiliadi DA, Markou M, Evangelatou E, Tzanela M, Tsagarakis S. Su- prasellar extension independently predicts preoperative pituitary hormone deficiencies in patients with nonfunctioning pituitary macroadenomas: a single-center experience. Hormones (Athens). 2020;19(2):245-251. doi: 10.1007/s42000-020-00183-0.

10. Zhang R, Wang Z, Gao L, Guo X, Feng C, Deng K, Lian W, Yao Y, Feng M, Bao X, Wang R, Xing B. Clinical Characteristics and Postoperative Recovery of Hypopituitarism in Patients with Nonfunctional Pituitary Adenoma. World Neurosurg. 2019;126:e1183-e1189. doi: 10.1016/j. wneu.2019.03.062.

11. Fleseriu M, Bodach ME, Tumialan LM, Bonert V, Oyesiku NM, Patil CG, Litvack Z, Aghi MK, Zada G. Congress of Neurological Surgeons Systematic Review and Evidence-Based Guideline for Pretreatment Endocrine Evaluation of Patients With Nonfunctioning Pituitary Adenomas. Neurosurgery. 2016;79(4):E527-E529. doi: 10.1227/NEU.0000000000001387.

12. Araujo-Castro M, Pascual-Corrales E, Acitores Cancela A, García Duque S, Ley Urzaiz L, Rodríguez Berrocal V. Status and clinical and radiological predictive factors of presurgical anterior pituitary function in pituitary adenomas. Study of 232 patients. Endocrine. 2020;70(3):584-592. doi: 10.1007/s12020-020-02455-6.

13. Yeliosof O, Gangat M. Diagnosis and management of hypopituitarism. Curr Opin Pediatr. 2019;31(4):531-536. doi: 10.1097/ MOP.0000000000000779. 
14. Jasim S, Alahdab F, Ahmed AT, Tamhane S, Prokop L, Nippoldt TB, Hassan Murad M. Mortality in adults with hypopituitarism: a systematic review and meta-analysis. Affiliations
Expand Endocrine. 2017;56(1):33-42. doi: 10.1007/s12020-016-1159-3.

15. Lee IH, Miller NR, Zan E, Tavares F, Blitz AM,

Sung $H$, Yousem DM, Boland MV. Visual De- fects in Patients With Pituitary Adenomas: The Myth of Bitemporal Hemianopsia. Am J Roentgenol. 2015;205(5):W512-W518. doi: 10.2214/ AJR.15.14527.

\section{The prevalence rate of hypopituitarism in patients with pituitary macroadenomas with various hormonal activities}

\author{
Yu.G. Krivosheeva' • I.A. Ilovayskaya'
}

Rationale: According to the recent epidemiological studies, prevalence of pituitary tumors amounts to 1 per 865 to 2688 adults. The prevalence rates of hypopituitarism in pituitary macroadenomas are highly variable and comprise $37 \%$ to $85 \%$ among newly diagnosed non-functioning pituitary adenomas (NFPA). There is virtually no data on the prevalence of hypopituitarism in the cohort of newly diagnosed macroadenomas secreting prolactin and somatotropic hormone.

Aim: To assess the prevalence of hypopituitarism in patients with pituitary macroadenomas with various hormonal activities and to identify its potential risk factors.

Materials and methods: We analyzed data from 293 patients with pituitary macroadenomas with various hormonal activities: NFPA $(n=121)$, prolactinomas $(n=59)$, and somatotropinomas $(n=113)$. The patients had been examined before any treatment was commenced.

Results: The prevalence rate of hypopituitarism was 59/121 (39.9\%) among the patients with NFPA, $18 / 59(27.3 \%)$ among those with prolactinomas, and 19/113 (14\%) among those with somatotropinomas $(p<0.001)$. The symptoms of chiasmal compression and chiasmal syndrome were significantly more prevalent in the patients with hypopituitarism, than in those without it in all subgroups. The relative risk of hypopituitarism in the patients with chiasmal compression was 2.10 for NFPA (95\% confidence interval (CI) 1.50-2.95, $\mathrm{p}=0.003), 1.667$ for prolactinomas $(95 \% \mathrm{Cl} 1.29$ $2.18, p=0.005)$, and 1.45 for somatotropinomas (95\% Cl 1.56-2.48, $\mathrm{p}=0.001)$. The relative risk of hypopituitarism in the patients with chiasmal syndrome was 1.66 for NFPA (95\% Cl 1.26-2.18, $\mathrm{p}=0.009), 2.08$ for prolactinomas $(95 \% \mathrm{Cl} 1.60$ 2.69, $p=0.001$ ), and 1.97 for somatotropinomas (95\% Cl 1.56-2.48, $\mathrm{p}=0.005)$. The NFPA subgroup had the highest rate of hypothyroidism (36/59, $61.0 \%$ ), whereas the prolactinoma subgroup had the highest rate of secondary hypogonadism $(34 / 59,57.6 \%)$; however, hypogonadism is one of the manifestations of persistent pathological hyperprolactinemia typical for prolactinomas. In the somatotropinoma subgroup, secondary hypothyroidism was found in $11 / 19$ (57.9\%) of the patients. Conclusion: Identification of hypopituitarism before any treatment depended on the type of hormonal activity of a pituitary tumor and was most frequent in NFPA. The risk factors for hypopituitarism were chiasmal compression, chiasmal syndrome, vertical diameter of the pituitary tumor, and its volume. Secondary adrenal insufficiency, being the most hazardous component of hypopituitarism, manifested most rarely.

Key words: pituitary macroadenoma, non-functioning pituitary tumors, prolactinomas, somatotropinomas, hypopituitarism

For citation: Krivosheeva YuG, Ilovayskaya IA The prevalence rate of hypopituitarism in patients with pituitary macroadenomas with various hormonal activities. Almanac of Clinical Medicine. 2021;49(4):261-267. doi: 10.18786/2072-0505-202149-047.

Received 20 October 2021; revised 29 October 2021; accepted 3 November 2021; published online 10 November 2021

\section{Conflict of interests}

The authors declare no conflict of interests.

\section{Authors' contributions}

Both authors have equally contributed to the manuscript, have read and approved the final version of the manuscript before submission, agreed to be accountable for all aspects of the work in ensuring that questions related to the accuracy or integrity of any part of the work have been appropriately investigated and resolved.

\section{Acknowledgements}

The authors would like to acknowledge A.A. Glazkov, MD, PhD, Research Fellow, Laboratory of Medical Physical Studies, MONIKI, for his assistance in the statistical analysis, G.A. Stashuk, MD, PhD, Department of Radiation Computed Tomography and Magnetic Resonance Imaging, Yu.N. Bardeeva, ophthalmologist, for their assistance in data collection for the study.
Yulia G. Krivosheeva - Research Fellow, Department of Therapeutic Endocrinology ${ }^{1}$; ORCID: https://orcid.org/0000-0002-2061-5235 $\triangle 61 / 2$ Shchepkina ul., Moscow, 129110, Russian Federation. Tel.: +7 (916) 4706041 E-mail:yulya.k.89@inbox.ru

Irena A. Ilovayskaya - MD, PhD, Associate Professor, Leading Research Fellow, Department of Therapeutic Endocrinology; Professor, Course of Special Endocrinology, Chair of Endocrinology, Postgraduate Training Faculty'; ORCID: https://orcid org/0000-0003-3261-7366. Tel.: +7 (916) 6772645. E-mail: irena.ilov@yandex.ru

'Moscow Regional Research and Clinical Institute (MONIKI); 61/2 Shchepkina ul., Moscow, 129110 Russian Federation 